\title{
Duodenal and Biliary Obstruction due to Extrinsic Compression by Recurrent Lobular Breast Carcinoma: A Case Report
}

\author{
Rukaiya Bashir Hamidua ${ }^{a}$ Bilal Asifa ${ }^{a}$ Harish Lavu $^{\text {b }}$ Thomas Kowalski $^{c}$ \\ Daniel P. Silver ${ }^{d}$ \\ aDepartment of Internal Medicine, Thomas Jefferson University Hospital, Philadelphia, PA, \\ USA; ${ }^{b}$ Department of Surgery, The Jefferson Pancreas, Biliary, and Related Cancer Center, \\ Thomas Jefferson University, Philadelphia, PA, USA; 'Department of Gastroenterology and \\ Hepatology, Thomas Jefferson University Hospital, Philadelphia, PA, USA; dDepartments \\ of Medical Oncology and Cancer Biology, Sidney Kimmel Cancer Center, Thomas Jefferson \\ University, Philadelphia, PA, USA
}

\section{Keywords}

Lobular breast carcinoma - Biliary · Obstructive jaundice · Duodenal obstruction .

Chemotherapy

\begin{abstract}
Metastases to the gastrointestinal tract (GIT) from breast carcinoma are rare, detected in approximately $<5 \%$ of all breast cancer patients. Invasive lobular carcinoma (ILC) is the most common histological type of breast cancer to metastasize to the GIT. We report a case of abdominal recurrence of ILC of the breast causing intra-abdominal contracture leading to extrinsic compression of the duodenum and periampullary biliary tree. Four years after the patient's diagnosis of a left breast pT1c, pN2, cM0 invasive lobular breast cancer, she presented with liver function tests consistent with biliary obstruction, and there was concern for a periampullary malignancy. Definitive diagnosis was achieved at laparotomy. This case demonstrates the importance of considering metastatic breast cancer as a potential cause of GI symptoms and radiological abnormalities affecting any part of the GIT of women with a previous history of lobular breast cancer. This case also highlights the effectiveness of chemotherapy in improving the survival and quality of life of these patients. Early recognition of this scenario enables prompt initiation of systemic therapy and avoids unnecessary surgical
\end{abstract}


Bashir Hamidu et al.: Duodenal and Biliary Obstruction due to Extrinsic

CompressionDuodenal and Biliary Obstruction due to Extrinsic Compression by

Recurrent Lobular Breast Carcinoma: A Case Report

treatment. Despite the rarity, such patients will be encountered in clinical practice given the high prevalence of breast cancer. Moreover, the fact that the presenting symptoms of GI metastasis from breast cancer are usually not specific to the origin and mimic a primary intestinal disorder, health-care professionals beyond oncologists, especially gastroenterologists and primary care physicians, should be aware of this entity.

(c) 2021 The Author(s).

Published by S. Karger AG, Basel

\section{Introduction}

Breast cancer is the second most common cancer among women in the USA [1]. The burden of breast cancer underscores the need to understand disease presentation and pattern of metastases. Breast cancer most commonly metastasizes to the bones, liver, and brain [2-4]. Gastrointestinal tract (GIT) metastases are rare, detected in approximately $<5 \%$ of all breast cancer patients [4-6]. The most frequent site of GI involvement by metastatic breast cancer is the stomach $[3,5]$. Invasive lobular carcinoma (ILC), the second most common histologic subtype of breast cancer, is more likely to metastasize to the GIT than is the more common subtype, invasive ductal carcinoma [2-4,6].

Metastatic breast cancer involving the GIT can produce a wide range of clinical and radiological presentations, often mimicking other gastrointestinal disorders and prompting referral to gastroenterology. As might be expected, there is a relative lower awareness of this condition in gastroenterology and primary care than oncology. This highlights the importance of considering metastatic breast cancer as a potential cause of GI symptoms and radiological abnormalities affecting any part of the GIT of women, especially those with a previous history of lobular breast cancer. Reported herein is the case of a 65-year-old female with recurrent ILC in the abdomen causing intra-abdominal and retroperitoneal contracture.

\section{Case Report/Case Presentation}

A 65-year-old female obstetric nurse was first referred to our gastroenterology center for further management of obstructive transaminitis. The patient has a medical history of a $2.0 \mathrm{~cm}$ left breast estrogen receptor (ER)-positive, progesterone receptor-positive, and human epidermal growth factor 2-negative ILC diagnosed 4 years prior, with 7 out of 9 ipsilateral lymph nodes involved with cancer. She was treated with lumpectomy, chemotherapy using dose-dense doxorubicin and cyclophosphamide (AC) followed by paclitaxel (T), wholebreast radiotherapy, and was placed on hormonal therapy with anastrozole. Three years after her initial breast cancer diagnosis, she had an onset of abdominal bloating, fatigue, and early satiety that progressed, leading her to see her primary care physician (PCP). At this time, diagnostic work up including computerized tomography (CT) of the abdomen and pelvis using intravenous contrast was unremarkable. Symptoms resolved but later recurred with associated significant fatigue, nausea, occasional vomiting and decreased PO tolerance, leading her to return to her PCP 6 months after the initial onset of symptoms. Blood test results were unremarkable (Table 1). Her PCP referred her for esophagogastroduodenoscopy (EGD) and colonoscopy which she completed 2 months later, and these studies were also unremarkable. She was subsequently treated for lactose intolerance with minimal improvement. She presented again 13 months after initial symptoms with similar complaints, and blood tests 
Bashir Hamidu et al.: Duodenal and Biliary Obstruction due to Extrinsic

CompressionDuodenal and Biliary Obstruction due to Extrinsic Compression by

Recurrent Lobular Breast Carcinoma: A Case Report

Table 1. Patient's laboratory results

\begin{tabular}{llll}
\hline Component name & $\begin{array}{l}6 \text { months after initial } \\
\text { symptom onset }\end{array}$ & $\begin{array}{l}13 \text { months after initial } \\
\text { symptom onset }\end{array}$ & Reference range \\
\hline Sodium & 144 & 144 & $134-144 \mathrm{mmol} / \mathrm{L}$ \\
Potassium & 4.1 & 4.4 & $3.5-5.2 \mathrm{mmol} / \mathrm{L}$ \\
Chloride & 103 & 104 & $97-108 \mathrm{mmol} / \mathrm{L}$ \\
CO2 & 25 & 25 & $18-29 \mathrm{mmol} / \mathrm{L}$ \\
BUN & 11 & 11 & $8-27 \mathrm{mg} / \mathrm{dL}$ \\
Glucose & 99 & 100 & $65-99 \mathrm{mg} / \mathrm{dL}$ \\
Calcium & 9.1 & 9.2 & $8.7-10.3 \mathrm{mg} / \mathrm{dL}$ \\
Total protein & 6 & 5.8 & $6.0-8.5 \mathrm{~g} / \mathrm{dL}$ \\
Albumin & 4.1 & 3.9 & $3.6-4.8 \mathrm{~g} / \mathrm{dL}$ \\
Total bilirubin & 0.5 & 0.7 & $0.0-1.2 \mathrm{mg} / \mathrm{dL}$ \\
AST & 20 & $\mathbf{1 3 8}(\mathbf{H})$ & $0-40 \mathrm{IU} / \mathrm{L}$ \\
ALT & 16 & $\mathbf{2 2 0}(\mathbf{H})$ & $0-32 \mathrm{IU} / \mathrm{L}$ \\
Alkaline phosphatase & 64 & $\mathbf{5 0 7}(\mathbf{H})$ & $39-117 \mathrm{IU} / \mathrm{L}$ \\
CA 19-9 & & $\mathbf{3 9}(\mathbf{H})$ & $<34 \mathrm{U} / \mathrm{mL}$ \\
\hline
\end{tabular}

Fig. 1. Contrast-enhanced CT scan of the abdomen in coronal view showing the "doubleduct" sign (double arrows) with the solid-line arrow representing a dilated CBD and the broken-line arrow representing a dilated MPD. Also shown are dilated intrahepatic ducts (single solid-line arrow). CT, computerized tomography; CBD, common bile duct; MPD, main pancreatic duct.

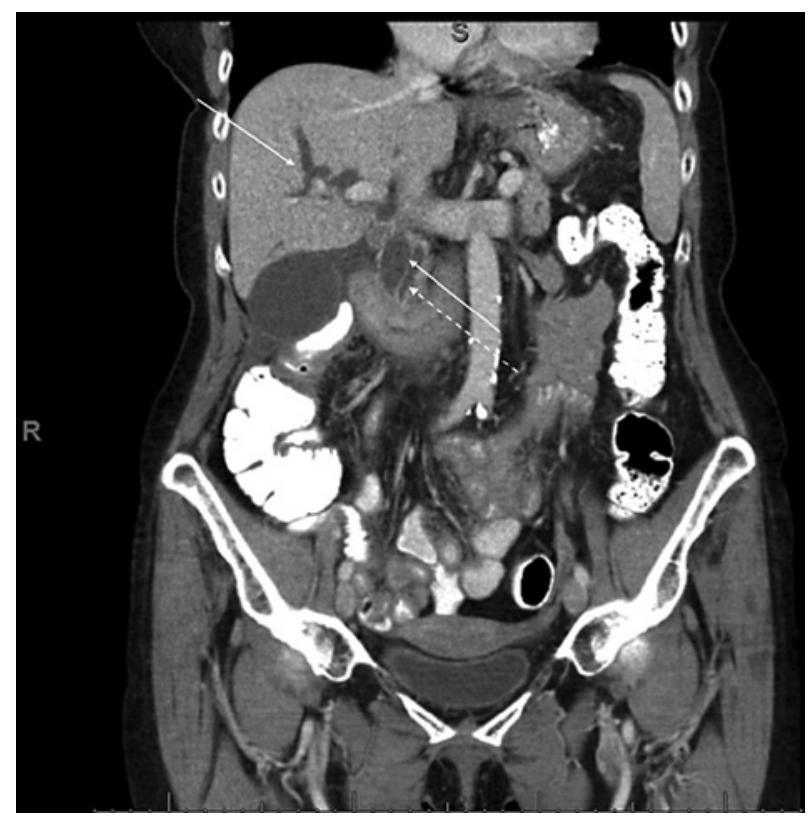

obtained were remarkable for elevated liver function tests compared to 6 months prior (Table 1), and cancer antigen 19-9 at $39(\mathrm{nl}<34 \mathrm{U} / \mathrm{mL})$. CT scan of the abdomen showed severe intra- and extrahepatic biliary ductal dilatation with the "double-duct" sign (common bile duct was $19 \mathrm{~mm}$ and main pancreatic duct was $4 \mathrm{~mm}$ ) without an identifiable cause (Fig. 1). Additionally, there was pericholecystic fluid without other gallbladder abnormalities and, colonic thickening at the hepatic flexure, with otherwise normal bowel caliber; the retroperitoneum, abdominal wall, and lymph nodes were normal. The patient was then referred to our gastroenterology center for management of obstructive transaminitis. 
EGD with endoscopic ultrasound (EUS) and colonoscopy was performed. EGD showed a stricture in the second portion of the duodenum (D2) that appeared to be extrinsic. On EUS, the mucosal layer of D2 appeared thickened and a fine-need aspiration (FNA) specimen of this segment was obtained. Cytopathologic examination of the FNA specimen showed clusters of atypical cells in the background of duodenal epithelial cells and inflammation. Additionally, on EUS, dilation of the common bile duct and main pancreatic duct without evidence of an obstructing lesion was reaffirmed, and the ampulla and pancreas appeared normal. The colonoscopy results were unremarkable. A follow-up magnetic resonance imaging (MRI) of the abdomen with intravenous contrast was performed, and it also reaffirmed the previous CT and endoscopic findings but additionally showed gallbladder dilation with no evidence of cholelithiasis or choledocholithiasis. The differential diagnosis given by the radiologist reading the MRI included a distal ductal/ampullary stricture, a neoplasm including ampullary carcinoma and cholangiocarcinoma, or an occult pancreatic mass. The patient was then referred to pancreatic surgery.

Given her elevated cancer antigen 19-9 and the cellular atypia observed in the endoscopic FNA specimen from the duodenum, the patient was presumed to have a local periampullary cancer. She was then recommended a pancreaticoduodenectomy (Whipple procedure). At laparotomy, there were several unexpected abnormalities noted upon abdominal entry. The stomach and gallbladder were distended. The small bowel mesentery in its entirety was indurated, foreshortened, and had numerous nodules. One of the nodules was biopsied and returned a benign lymph node. The duodenum was adherent to the retroperitoneum, and the transverse colon was adherent to the head of the pancreas. The right colon was adherent to the abdominal wall and also indurated. Based on the extent of the abdominal findings, the surgeons concluded that disseminated malignancy was present and the Whipple procedure was aborted. No bypasses were performed to the patient's bile duct or the stomach because the small bowel mesentery was so foreshortened that it would not allow reach into the upper abdomen above the colon. Her gallbladder was then resected, and a partial omentectomy was also performed to provide additional tissue for pathologic examination. Histopathological examination results of specimens obtained from the surgery revealed findings consistent with metastatic lobular breast carcinoma (Fig. 2). These results were confirmed by immunohistochemical staining (Fig. 2) which additionally, demonstrated that the cancer cells were $\mathrm{ER}+$, progesterone receptor-, and human epidermal growth factor 2+. Postoperatively, a percutaneous transhepatic biliary drain was placed by interventional radiology which relieved her biliary obstruction after failure of biliary stenting with endoscopic retrograde cholangiopancreatography; the duodenoscope could not traverse the stricture in D2 despite sequential dilation with a wire-guided dilation balloon.

After discharge from the hospital, the patient followed up with medical oncology. Anastrozole was discontinued and she received 2 months of weekly Taxol without significant response. Intercurrent with this, she was discovered to have a large intra-abdominal abscess near her percutaneous transhepatic biliary drain. The abscess was drained by interventional radiology. She was subsequently started on Fulvestrant and Palbociclib 2- and 4- months after her surgery, respectively. Significant disease regression occurred, all drains were removed, and she enjoyed an active lifestyle with full functional status. After 3 and half years of Fulvestrant and Palbociclib, she presented with left eye irritation. This progressed to diplopia with abnormal extraocular movements on exam 59 months after the initial onset of symptoms. MRI showed a large left retro-orbital mass, and a biopsy was consistent with her lobular breast cancer. Palbociclib and Fulvestrant were discontinued. She was radiated with external beam radiation therapy to the left eye. An analysis of ctDNA showed autonomously signaling ESR1 mutations. She is now enrolled in a trial that involves a new highly active SERD with Abemaciclib and remains well.

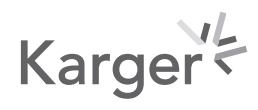



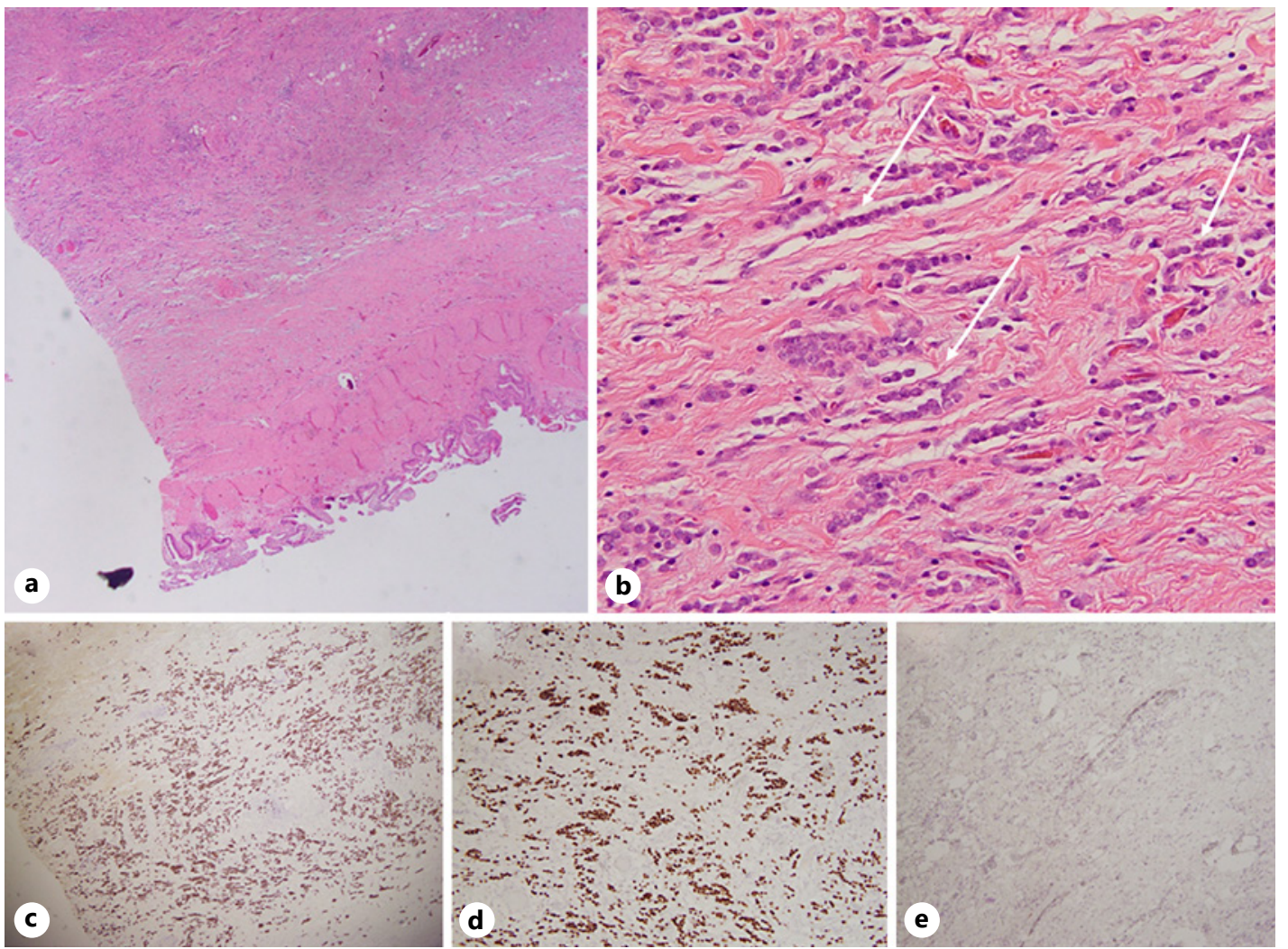

Fig. 2. Histopathological examination of the excised gallbladder. $\mathbf{A} \times 2$ magnification image showing a normal gallbladder mucosa (b). A $\times 20$ magnification image showing a section of the gallbladder infiltrated by metastatic lobular carcinoma. Arrows are pointing to the "single file" arrangement of tumor cells in a fibrous stroma, which is considered to be highly characteristic of infiltrating lobular carcinoma of the breast. Immunohistochemical staining of the tumor cells are shown in images C, D, and E. Carcinoma of the breast is supported by positive AE1/AE3 (c) and GATA3 stains (d). GATA3 is highly specific for carcinoma of breast origin. Furthermore, lobular-subtype of breast carcinoma is supported by the loss of E-cadherin in tumor cells as evidenced by the absence of E-cadherin staining (e).

\section{Discussion/Conclusion}

We report an instance of recurrence of ILC of the breast in the abdomen. The patient presented with obstructive pattern transaminitis, and her preoperative diagnostic testing was concerning for a periampullary malignancy. However, laparotomy revealed gallbladder metastasis that was clinically silent and metastatic ILC causing intra-abdominal contracture, with consequent extrinsic compression of the duodenum and periampullary biliary tree. This presentation is similar to the first description of Ormond disease in the 1940s in which a patient presented with obstructive uropathy and exploratory laparotomy showed extensive retroperitoneal fibrosis with resultant compression of the ureters revealed $[7,8]$.

Most cases of duodenal obstruction by a malignancy are due to direct invasion by tumor cells or from an ampullary or pancreatic head mass [2]. Although rare, bowel obstruction by extrinsic compression from a fibrotic process induced by a malignancy has been reported, and similar to our case, definitive diagnosis was only established at laparotomy $[9,10]$. Similarly, extrahepatic biliary obstruction caused by metastatic breast carcinoma is also rare and in the few reported cases, biliary obstruction was often related to metastatic periductal or peripancreatic LAD, or direct metastases to the bile ducts or pancreas [11]. 
The proposed mechanism of the metastatic pattern of ILC is thought to result from loss of expression of membrane E-cadherin, and commonly lack fibronectin, resulting in loose binding between cells $[6,8]$. This leads to absent cell to cell adhesion, which facilitates permeation through tissue planes, causing diffuse disease [3]. This pattern contrasts with ductal carcinomas, which tend to produce nodular lesions [3]. The fibrosis and contracture caused by metastatic ILC are thought to be secondary to the intense reaction invoked by the characteristic "single file" pattern of tissue infiltration by the tumor cells $[4,12]$. Single file is a pattern that can be seen on cytological or histological examination in which tumor cells are seen arranged in linear rows which appear as if one is following the footsteps of another (Fig. 2b) [12].

The diagnostic modalities most often used to identify metastatic disease to the GIT are CT of the abdomen and pelvis and EUS with or without FNA. The literature, however, demonstrates difficulty in diagnosing metastatic spread of breast cancer to the GIT [5]. Autopsy studies show metastatic lobular breast carcinoma cases had a high potential for misinterpretation, possibly because most metastases consisted of infiltrating strands of pleomorphic cells without nodule formation [13]. Other diagnostic challenges include that these patients tend to not be very symptomatic for long periods of time, and if symptoms are present, they are often nonspecific. Also, as presented here, GIT metastases from ILC often occur many years after the diagnosis of the primary tumor, leading clinicians not to consider the possibility of recurrence. The disease-free interval between primary breast cancer and GIT metastases may range from synchronous to as long as 30 years, and occasionally the GIT manifestation precedes the diagnosis of breast cancer $[3,14]$. An additional challenge in our case was the low sensitivity of preoperative imaging to pick up additional metastatic sites and the limitation of endoscopy in providing diagnosis given that the disease was beyond the mucosa. This patient's endoscopy revealed a duodenal stricture and suggested extrinsic compression. The FNA obtained from this stricture showed cellular atypia. Given this information, in retrospect, it may have been useful to probe further for metastatic breast cancer with an additional modality such as positron emission tomography (PET)/CT and to have stained this specimen for the ER. PET with 18F-fluorodeoxyglucose and PET/CT in breast carcinoma is useful in accurately staging the metastatic disease, assessing response to systemic treatment, and clarifying equivocal findings on other imaging [15]. It is possible that immunohistochemical staining for ER or PET/CT could have detected the widely metastatic disease in our patient, sparing her a laparotomy.

Systemic treatment with chemotherapy, endocrine therapy, or both are usually employed in patients with metastases to the GIT since patients usually present with diffuse involvement of multiple organs [3]. Previous reports state that the median overall survival after a diagnosis of GI metastasis is 16 months (range, 5-41 months), which is lower than the median survival of all women with metastatic disease secondary to breast cancer (range, 24-36 months) [3]. However, as seen in this case, current therapies have had a significant impact on the mortality and survival rates of breast cancer.

In conclusion, this case demonstrates an unusual presentation of obstructive transaminitis and duodenal obstruction from extrinsic compression caused by retroperitoneal infiltration with metastatic lobular breast carcinoma. Notably, such metastases from ILC can present years after initial diagnosis because breast cancer can have prolonged dormancy. A high level of suspicion for metastases should be maintained when patients with a history of lobular breast cancer present with GI symptoms, or with a presumed new primary tumor or stricture of the GIT. Furthermore, given the prevalence of breast cancer and the fact that the presenting symptoms of GI metastasis from breast cancer are usually not specific to the origin and mimic a primary intestinal disorder [14], health-care professionals beyond oncologists, especially gastroenterologists and PCPs, may such encounter patients. Also, the central role

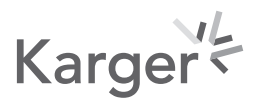




\begin{tabular}{l|l}
\hline Case Rep Gastroenterol 2021;15:869-876 \\
\hline DOI: 10.1159/000518874 & $\begin{array}{l}\text { (c) 2021 The Author(s). Published by S. Karger AG, Basel } \\
\text { www.karger.com/crg }\end{array}$ \\
\hline
\end{tabular}

Bashir Hamidu et al.: Duodenal and Biliary Obstruction due to Extrinsic

CompressionDuodenal and Biliary Obstruction due to Extrinsic Compression by

Recurrent Lobular Breast Carcinoma: A Case Report

of endoscopy and biopsy in ascertaining the diagnosis of such patients additionally highlights the importance of awareness of this presentation by gastroenterologists. Even though the usefulness of endoscopy in making a diagnosis may be limited if the obstruction is extrinsic and does not involve the mucosa [3]. The effectiveness of chemotherapy in improving the survival of patients as displayed here underlies the importance of early recognition which would enable prompt initiation of systemic therapy, thus helping to avoid unnecessary prolonged diagnostic testing and surgical treatment.

\section{Statement of Ethics}

The subject of this case report has provided written informed consent for publication of the case. The need for approval was waived by the Institutional Review Board at the Thomas Jefferson University, as this was a single-subject case report.

\section{Conflict of Interest Statement}

The authors have no conflicts of interest to declare.

\section{Funding Sources}

The authors disclose no sponsorship or funding arrangements relating to their research.

\section{Author Contributions}

The initial version of the manuscript was prepared by Rukaiya Bashir Hamidu. Bilal Asif, Thomas Kowalski, and Harish Lavu edited the manuscript and provided intellectual input. Daniel P. Silver edited and proofread the manuscript, reviewed the data, and provided intellectual input. All the authors approved the final version of the manuscript.

\section{Data Availability Statement}

The authors confirm that all data generated or analyzed during this study are included in this article. Further enquiries can be directed to the corresponding author.

$\mathrm{CA}$, cancer antigen.

\section{References}

1 Group USCSW. U.S. cancer statistics data visualizations tool, based on 2019 submission data (1999-2017). USA: Department of Health and Human Services, Centers for Disease Control and Prevention and National Cancer Institute; June 2020. Available from: www.cdc.gov/cancer/dataviz.

2 Daniels IR, Layer GT, Chisholm EM. Lessons to be learned: a case study approach: bowel obstruction due to extrinsic compression by metastatic lobular carcinoma of the breast. J R Soc Promot Health. 2002;122(1): 61-2.

3 Nazareno J, Taves D, Preiksaitis HG. Metastatic breast cancer to the gastrointestinal tract: a case series and review of the literature. World J Gastroenterol. 2006;12(38):6219.

4 Nikkar-Esfahani A, Kumar BG, Aitken D, Wilson RG. Metastatic breast carcinoma presenting as a sigmoid stricture: report of a case and review of the literature. Case Rep Gastroenterol. 2013;7(1):106-11.

\section{Karger's}


Bashir Hamidu et al.: Duodenal and Biliary Obstruction due to Extrinsic

CompressionDuodenal and Biliary Obstruction due to Extrinsic Compression by

Recurrent Lobular Breast Carcinoma: A Case Report

5 Zhao R, Li Y, Yu X, Yang W, Guo X. Duodenal metastasis from recurrent invasive lobular carcinoma of breast: a case report and literature review. Int J Clin Oncol. 2012;17(2):160-4.

6 Nihon-Yanagi Y, Park Y, Ooshiro M, Aoki H, Suzuki Y, Hiruta N, et al. A case of recurrent invasive lobular carcinoma of the breast found as metastasis to the duodenum. Breast Cancer. 2009;16(1):83-7.

7 Ormond JK. Bilateral ureteral obstruction due to envelopment and compression by an inflammatory retroperitoneal process. J Urol. 1948;59(6):1072-9.

8 Yousef GM, Gabril MY, Al-Haddad S, Mulligan AM, Honey RJ. Invasive lobular carcinoma of the breast presenting as retroperitoneal fibrosis: a case report. J Med Case Rep. 2010;4(1):175-4.

9 McCarthy JG, Porter MR, Veenema R. Retroperitoneal fibrosis and large bowel obstruction: case report and review of the literature. Ann Surg. 1972;176(2):199.

10 Schneider CF. Idiopathic retroperitoneal fibrosis producing vena caval, biliary, ureteral and duodenal obstructions. Ann Surg. 1964;159(2):316.

11 Franco D, Martin B, Smadja C, Szekely AM, Rougier P. Biliary metastases of breast carcinoma. The case for resection. Cancer. 1987;60(1):96-9.

12 Pandiar D, Smitha T. "Single file" pattern in histopathology. J Oral Maxillofac Pathol. 2020 May-Aug;24(2):204.

13 Washington K, McDonagh D. Secondary tumors of the gastrointestinal tract: surgical pathologic findings and comparison with autopsy survey. Mod Pathol. 1995;8(4):427-33.

14 Khairy S, Azzam A, Mohammed S, Suleman K, Khawaji A, Amin T. Duodenal obstruction as first presentation of metastatic breast cancer. Case Rep Surg. 2015;2015:605719.

15 Constantinidou A, Martin A, Sharma B, Johnston SR. Positron emission tomography/computed tomography in the management of recurrent/metastatic breast cancer: a large retrospective study from the Royal Marsden Hospital. Ann Oncol. 2011;22(2):307-14. 